making. Myocardial fractional flow reserve $\left(\mathrm{FFR}_{\text {myo }}\right)$ is a special index of the functional severity of coronary stenosis. Although the application of $\mathrm{FFR}_{\text {myo }}$ to assess intermediate coronary lesion is widely performed in some centers in developed countries, its use in China was lagged. Because it is relatively expensive to measure $\mathrm{FFR}_{\text {myo, }}$ it will be beneficial to save the expenses and to short operation time if CAG and IVUS criteria could be clinically used as tools to discriminate functional significant of intermediate stenosis.

Materials and Methods In 46 lesions of intermediate severity (eg, $40 \%-60 \%$ diameter stenosis) we assessed 1 . by pressure wire: myocardial fractional flow reserve $\left(\mathrm{FFR}_{\mathrm{myo}}\right.$, index of functional significance), and 2. by IVUS: minimal lumen cross-sectional area (MLA) and percent area stenosis at the lesion site. Receiver operating characteristic (ROC) curve analysis was performed to establish the best cut-off values of IVUS indexes (ie, MLA and percent area stenosis) that were most predictive of $\mathrm{FFR}_{\text {myo }}<0.75$.

Results FFRmyo in 46 lesions of angiographic intermediate stenosis $(49 \pm 11 \%)$ was significantly lower than it was in angiographic normal artery $(0.83 \pm 0.15$ vs $0.97 \pm 0.02, p<0.01)$. Fourteen lesions $(30 \%)$ were functionally critical (eg, $\mathrm{FFR}_{\mathrm{my}}<0.75$ ). By regression analysis, percent area stenosis had a significant inverse correlation with $\mathrm{FFR}_{\text {myo }}$ $(\mathrm{r}=-0.68, \mathrm{p}<0.01)$. MLA showed a significant positive relation with $\mathrm{FFR}_{\text {myo }}(\mathrm{r}=0.63, \mathrm{p}<0.01)$. By ROC analysis, we identified an IVUS area stonosis $\geq 65 \%$ (sensitivity $100 \%$, specificity $72 \%$ ), a minimal lumen cross-sectional area $\leq 4 \mathrm{~mm}^{2}$ (sensitivity $93 \%$, specificity $77 \%$ ) to be the best cut-off values to fit with $\mathrm{FFR}_{\text {myo }}<0.75$.

Conclusion IVUS area stenosis $\geq 65 \%$ and minimal lumen crosssectional area $\leq 4 \mathrm{~mm}^{2}$ reliably identified functionally critical intermediate coronary stenosis.

\section{e0528 IMPROVEMENT IN DOOR-TO-BALLOON TIMES IN PATIENTS WITH ST-ELEVATION MYOCARDIAL INFARCTION IN BEIJING ANZHEN HOSPITAL}

doi:10.1136/hrt.2010.208967.528

Song Li, Yan Hongbing. Beijing Anzhen Hospital

Background Guidelines recommend a door-to-balloon time (D2B)< 90 min for patients with ST-segment elevation myocardial infarction (STEMI) who undergo primary percutaneous coronary intervention (PPCI). Little is currently reported about systems of care to achieve this $\mathrm{D} 2 \mathrm{~B}$ performance in China. The objective of this study was to compare median $\mathrm{D} 2 \mathrm{~B}$ time and the percentage of patients treated within $90 \mathrm{~min}$ in Beijing Anzhen Hospital during two different periods.

Methods STEMI protocol to shorten D2B time in Beijing Anzhen Hospital in recent 2 years included activation of the cardiac catheterisation laboratory by the cardiologist in emergency department; all members of the intervention team use cell phones; catheterisation laboratory staff arrival within $20-30 \mathrm{~min}$ of activation; enhancing close integration of ambulance networks and hospital such as advance notification and pre-hospital ECG by ambulance crews; and bypassing the emergency room. Data were prospectively collected from STEMI patients admitted within $12 \mathrm{~h}$ of symptom onset and underwent PPCI during two different periods: in 2006 $(n=138)$ and in $2009(n=156)$. D2B times and its components were compared between two groups.

Results The median D2B time decreased from $120 \mathrm{~min}$ $\mathrm{IOR}=88-150 \mathrm{~min})$ in 2006 to $80 \mathrm{~min}(\mathrm{IOR}=60-105)$ in 2009 $(\mathrm{p}<0.0001)$. More precisely, subinterval time periods for the median door-to-ECG time decreased from $5 \mathrm{~min}$ in 2006 to $3 \mathrm{~min}$ in 2009 $(p<0.0001)$, the median door-to-sign the operation approval time decreased from $35 \mathrm{~min}$ in 2006 to $15 \mathrm{~min}$ in 2009 ( $p<0.0001)$, the median sign-to-puncture time decreased from $80 \mathrm{~min}$ to $60 \mathrm{~min}$ $(p<0.0001)$, and the median puncture-to-balloon decreased from
$25 \mathrm{~min}$ to $20 \mathrm{~min}(\mathrm{p}<0.0001)$. Importantly, the proportion of patients with D2B times $<90$ min increased from $21.4 \%$ in 2006 to $65.7 \%$ in $2009(\mathrm{p}<0.0001)$.

Conclusions D2B time decreased significantly from 2006 to 2009 in Beijing Anzhen Hospital. Accordingly, the percentage of patients treated within 90 min has dramatically increased from 2006 to 2009 Our experience demonstrates the effectiveness of process changes targeting timeliness of PPCI.

\section{e0529 THE EFFECT OF PRIMARY PCI OF CULPRIT ARTERY ON EPICARDIAL FLOW IN NONCULPRIT ARTERY IN PATIENTS WITH ANTERIOR STEMI}

doi:10.1136/hrt.2010.208967.529

Wang Jian, Yan Hongbing. Beijing Anzhen Hospital

Objective To study the effect of primary PCI of culprit artery on epicardial flow in Nonculprit Artery in patients with STEMI.

Methods Enrolled 401 Anterior STEMI patients underwent primary PCI and the culprit artery is LAD (the test group) enrolled 100 patients that Coronary artery angiography suggesting normal as the control group. To observe the differences of CTFC and MBG pre and post primary $\mathrm{PCI}$ in both culprit and nonculprit artery, and to observe CTFC and MBG in the control group.

Results Nonculprit artery (LCX)'sCTFC and MBG level in patients with Anterior STEMI were different from those in the control group before primary $\mathrm{PCI}$, and after primary $\mathrm{PCI}$, nonculprit artery (LCX)'sCTFC and MBG level were improved, but still not recovery to normal level.

Conclusion Nonculprit artery's perfusion May be impaired in patients with STEMI, nonculprit artery's perfusion May be improved after primary PCI, but still lower than those in the control group.

\section{e0530 LONG-TERM FOLLOW-UP AFTER DRUG-ELUTING STENTS FOR PATIENTS WITH ST-SEGMENT ELEVATION MYOCARDIAL INFARCTION: A SINGLE CENTER STUDY}

doi:10.1136/hrt.2010.208967.530

Zhao Yong, Yan Hongbing. Beijing Anzhen Hospital

Objective To evaluate the long-term efficacy and safety of DES in the setting of primary PCI in our single center.

Methods From September 2004 to November 2008, 464 patients undergoing primary PCI for STEMI were included at the 28th Division in Beijing Anzhen Hospital. The adverse events were compared among different types of DES.

Results The major adverse cardiac events (MACE, including sudden cardiac death, stent thrombosis, target lesion revascularization [TLR], target vessel revascularization [TVR], reinfarction and coronary artery bypass graft), all-cause death, major bleeding, antiplatelet therapy and secondary prevention of coronary heart disease of different types of DES were no significant differences. The cumulative incidence of MACE and stent thrombosis were $9.3 \%$ and $1.1 \%$ respectively, different types of DES were $9.4 \%, 5.1 \%, 5.9 \%$, $6.6 \%(p=0.483)$ and $3.1 \%, 0.0 \%, 0.0 \%, 0.0 \%(p=0.092)$ respectively. The patients in the DES group had longer duration of dual antiplatelet therapy (average $16.2 \pm 6.7$ months). No major bleeding occurred in all patients with the long-term and low-dose aspirin (100 mg).

Conclusions Different types of DES have the same results in the setting of primary PCI, and were long-term safe and effective with a reasonable strategy for anti-platelet therapy and secondary prevention. 